Cross-cultural equivalence of the Career Decision-Making Self-Efficacy Scale - Short Form: An Australian and South African comparison

Authors: $\quad$ Peter A. Creed, Griffith University - Gold Coast, Australia Wendy Patton, Queensland University of Technology, Australia Mark B. Watson, University of Port Elizabeth, South Africa

Contact: $\quad$ Dr Peter A. Creed

School of Applied Psychology

Griffith University - Gold Coast

PMB 50 Gold Coast Mail Centre

Gold Coast, Queensland

Australia, 9726

Telephone: +61755528881

Facsimile: $\quad+61755528291$

E-mail: $\quad$ p.creed@mailbox.gu.edu.au 


\title{
Cross-cultural equivalence of the Career Decision-Making Self-Efficacy \\ Scale - Short Form: An Australian and South African comparison
}

\begin{abstract}
The present study examined the reliability, content validity and cultural equivalence of the short form of the Career Decision Making Self-Efficacy Scale (CDMSES-SF: Betz, Klein, \& Taylor, 1996). In response to calls to conduct studies using the measure with high school age samples (Luzzo, 1996), data were gathered from two samples of high school students, one from Australia and one from South Africa. The findings were in accord with earlier studies in that they failed to find five factors. Three factors were found with each sample, however these factors were different in each sample, and different from those reported in samples of US college students, suggesting cross-cultural differences in the construct. The authors suggest that a more parsimonious version of the CDMSES-SF is possible, that the CDMSESSF does not adequately reflect its theoretical origins, and that cultural equivalence cannot be assumed.
\end{abstract}


Bandura's (1986) social cognitive theory proposes that individuals' beliefs and confidence in their ability to perform given tasks and behaviours successfully (i.e., their self-efficacy expectations) influence their choices, performance and persistence in these tasks and behaviours. Thus, while low self-efficacy expectations would lead to avoidance behaviour, high self-efficacy expectations would encourage approach behaviour towards specific tasks or behaviours. The concept of self-efficacy expectations suggests further that how individuals behave can be better predicted by their beliefs about their capabilities than by their actual capabilities. As such, self-efficacy has been recognised as one of the most theoretically, heuristically, and practically useful concepts formulated in modern psychology (Betz, Klein, \& Taylor, 1996).

The application of the self-efficacy construct to career behaviour was pioneered by Betz and Hackett (1981) who established that college students' beliefs about their educational and career capabilities were significantly related to the range and type of career options they considered. High levels of career decision-making self-efficacy should lead to increased participation in career decision-making tasks and behaviours, while low levels of career decision-making self-efficacy would lead to the avoidance of such activities. Subsequent research has established the utility of career self-efficacy as a predictor of career exploration (Blustein, 1989), career maturity (Luzzo, 1995), and stability in the career patterns of college students (Gianakos, 1999). Most career self-efficacy research has focused on tertiary students, however, and has failed to heed the call to extend the developmental "searchlight" (Lent \& Hackett, 1987, p.372) towards earlier developmental levels. Nevertheless, metaanalyses and reviews have continued to endorse the utility of the career self-efficacy construct in career theory (Betz \& Voyten, 1997; Hackett \& Lent, 1992), career counselling interventions (Solberg et al., 1994), and as a predictor of career decision-making intentions and behaviours (Gianakos, 1999; Lent, Brown, \& Hackett, 1994). 
While there seems to be general consensus about the utility of the construct of career selfefficacy, development of appropriate tools to measure it has proved more problematic. Osipow (1991) has referred to many such measures as "homemade, unvalidated, of marginal or unknown reliability" (p. 325). Taylor and Betz (1983) were the first to develop a 50-item standardized measure of career decision-making self-efficacy. The rational structure of their Career Decision-Making Self-Efficacy Scale's (CDMSES) five subscales reflects the career choice competencies that Crites (1961) proposed as relevant for the career decision-making process, that is, accurate self-appraisal, gathering occupational information, goal selection, making plans for the future, and problem solving.

Taylor and Betz's (1983) principal components factor analysis of the CDMSES failed to support the five factors they proposed. Subsequent factor analyses of the CDMSES have reported similar findings (Peterson \& Delmas, 1998; Robbins, 1985; Taylor \& Popma, 1990), leading to the suggestion that the CDMSES may be more appropriate as a generalized measure of career decision-making self-efficacy. There is some ambivalence about the latter suggestion. For instance, Betz, Klein, and Taylor (1996), while acknowledging the lack of psychometric support for a five-factor structure for the CDMSES, argue that this factor structure remains a "useful framework" (p. 56) for understanding the stages of career decision-making. Similarly, in developing a short form of the measure (CDMSES-SF: Betz, Klein \& Taylor, 1996), the authors emphasised the need to retain the rational structure of the original instrument for its theoretical and applied utility, even though this structure had not been supported by factor analyses.

The 25-item short form was developed by eliminating five of the ten items from each of the CDMSES subscales. This decision was based on four criteria: content specificity or narrowness, an item-subscale correlation equal to or above .50 , the item loading on the appropriate factor in the Taylor and Popma (1990) factor analysis, and a recommendation for 
retention on the basis of the split scale analysis by Gati, Osipow, and Fassa (1994). Thus, the CDMSES-SF consists of five 5-item subscales. Scale scores were computed by summing responses to each scale's items, and the total score was the sum of the scores of the five subscales.

Reliability and validity studies have been predominantly conducted on college age students. In addition, while a number of studies have published research findings on the CDMSES-SF, many have not reported reliability data (Betz \& Klein, 1996; Luzzo, Hitchings, Retish, \& Shoemaker, 1999), and have reported validity data only in the form of correlations between measures (Betz \& Klein; Gloria \& Hird, 1999). Studies using the CDMSES-SF that have reported reliability data have shown it to be highly reliable. Betz et al. (1996) reported subscale alphas ranging from 0.73 to 0.83 , and a total score alpha of 0.94 in a college sample. Betz and Voyten (1997), also with a college sample, reported internal reliability coefficients for the subscales ranging from .69 to .83 , and a total score coefficient of .93 . Gloria and Hird (1999) reported total score coefficients of .95 for white college students and .97 for racial/ethnic minority college students. Watson, Brand, Stead, and Ellis (2001) studied a sample of South African university students and reported only one subscale with an internal reliability below .70, and a total score coefficient of .91.

Validity evidence using the short form of the CDMSES is limited. Relationships in the expected directions have been reported between the CDMSES-SF and the Certainty and Indecision subscales of the Career Decision Scale (CDS: Osipow, 1987; Betz, Klein, \& Taylor, 1996) and the Vocational Identity Scale (Holland, Johnston, \& Asama, 1993). Betz and Klein (1996) reported relationships between career self-efficacy measures and generalized self-efficacy. Betz and Voyten (1997) reported separate correlations for females and males, with significant relationships between career self-efficacy and academic and career outcome expectations, exploratory intentions, and career indecision. 
To date, there have been no published psychometric studies on the CDMSES-SF on high school samples. Unpublished research by Albion (2000) with Australian high school students reported subscale alphas between .64 and .74, and a total score alpha of .90. Albion's data also illustrated negative correlations between CDMSES-SF scores and career difficulties and undecidedness, and positive relationships between CDMSES-SF and satisfaction and confidence in career choice. A significant relationship between self-efficacy for career related tasks and student confidence in answering questions about career related information was also found by Albion. Kraus and Hughey (1999) used the CDMSES-SF as one measure to evaluate the effectiveness of a high school intervention. No reliability data were reported and mixed findings on the relationship between CDMSE and career indecision were found.

Similar to the paucity of studies reporting on the psychometric aspects of the CDMSES-SF, there have been very few reported factor analyses. Two studies identified were conducted with college age samples. Betz et al. (1996) attempted a five factor and two factor solution following a principal components analysis. In the five-factor solution, these authors found only two strong factors, Occupational Information and Goal Selection, which also included Planning items. Factors 3 and 4 included Problem Solving and Self-Appraisal items, and the fifth factor was one sole Self-Appraisal item. In the two-factor solution, a Decision Making factor included Self-Appraisal, Planning and Goal Selection items, and an Information Gathering factor included Problem Solving as well as Occupational Information items. Watson et al. (2001) used a confirmatory factor analysis technique to determine whether the CDMSES-SF fit the five theorised subscales. These authors reported that the CFA model fitted the data poorly.

In relation to the factor analytic studies conducted with the CDMSES and the CDMSES-SF, three confounding factors are worth noting. First, all studies to date have utilised college level students. This raises questions as to how widely the results of such 
studies can be generalised, and how satisfactory the factor solutions are given the restricted range of responses likely to be found in this population. Studies need to be conducted with high school age students, an issue emphasised by Luzzo (1996). Second, studies to date have generally utilised orthogonal procedures to determine solutions. Orthogonal solutions operate to constrain the factors to be uncorrelated with one another, and are unlikely to provide the most adequate solutions when the factors are correlated. The rational basis for constructing the CDMSES and CDMSES-SF are likely to have produced related constructs (all subscales were constructed to measure confidence in different aspects of career decision-making), and substantial correlations among the items have been reported. For example, for the CDMSESSF, Watson, Brand, Ellis, and Stead (2001) reported item-total correlations with the full 25item scale ranging between .30 to .64 , with 18 of the 25 correlations being more than or equal to .50 . The third issue relates to the suitability of the CDMSES for cross-cultural assessment. It is common to assume that administrating tests across cultures that speak a common language will allow for comparability of test scores. However, comparability of test scores cannot be assumed unless tested, and this assumption is likely to reflect an example of the cultural equivalence fallacy (Helms, 1992).

Thus, the present study examines the content validity of the 25-item CDMSES-SF by the application of factor analysis procedures. First, the analyses are conducted on two samples of high school students, one sample of Australian high school students and one sample of South African high school students. This allows for an examination of the CDMSES-SF on samples other than college students, on samples more heterogeneous than college students, on samples from diverse cultures, and on cultures different from the original sample/s used to develop and validate the scale. Factor analytic techniques are the most widely used methods for evaluating cultural equivalence as they allow an analysis of the underlying dimensions of a scale across cultures. These techniques include exploratory factor analysis, the use of 
congruence coefficients, factor score correlations, and confirmatory factor analysis (BenPorath, 1990). This study commenced with exploratory factor analysis, and given the reported relatedness of the CDMSES-SF subscales, the analyses are conducted by applying oblique rotation to determine the factor analytic solution.

\section{Method}

\section{Participants}

The total number of participants included in the study was 979 secondary school students enrolled in Grades 8-12 across two secondary schools, one in the south-eastern part of Australia and the other in the eastern region of South Africa. There were $563(58 \%)$ students from Australia, consisting of $302(54 \%)$ females and 251 (46\%) males. These Australian students had a mean age of 15.45 years $(S D=1.44)$, with $110(20 \%)$ in Grade 8, $111(20 \%)$ in Grade 9, 122 (22\%) in Grade 10, 125 (22\%) in Grade 11, and 97 (17\%) in Grade 12. There were $416(42 \%)$ students from South Africa, consisting of 225 (54\%) females and 191 (46\%) males. The South African students had a mean age of 15.30 years $(S D=1.41)$, with $82(20 \%)$ in Grade 8, 95 (23\%) in Grade 9, 110 (26\%) in Grade 10, 93 (22\%) in Grade 11, and $36(9 \%)$ in Grade 12. The two schools selected were considered to be matched on socio-economic status and geographic grounds, that is, both schools were suburban based in medium sized cities, and each was established in a middle level socio-economic area of their respective cities.

\section{Instrument}

Participants completed the short form of the Career Decision-Making Self-Efficacy Scale (CDMSES-SF; Betz et al., 1996). Respondents were requested to indicate their level of 
confidence on a five-point scale, with endpoints of "no confidence at all" to "complete confidence". Although Betz et al. (1996) advocated a 10-level confidence continuum, this was modified to 5-levels for the present study as the longer continuum was deemed to be too complex for high school students. This gave a possible range for the 25 -item full scale of 25 125 , and for each of the subscales of 5-25, with higher scores indicating more confidence. Minor changes were made to a small number of items in the scale to better suit the Australian and South African samples. For example, the word "major", which is not in common use in Australia or South Africa, was omitted or changed to "career". The question, "Choose a major or career that will fit your interests?", became "Choose a career that will fit your interests?". Other sample items were, "How much confidence do you have that you could: choose a career that will fit your preferred lifestyle (Goal Selection), find information in the library about occupations that you are interested in (Information Gathering), change occupations if you are not satisfied with the one you enter (Problem Solving), make a plan of your goals for the next five years (Planning), and accurately assess your abilities (SelfAppraisal)". Consistently high internal reliability coefficients have been reported for the full 25-items and for each of the subscales. For example, Betz, Klein, and Taylor (1996) reported an internal reliability coefficient of .94 for the full scale, and coefficients ranging from .73 to .83 for the subscales. In the present study, for the Australian sample, the internal reliability co-efficient for the full 25 -items was .94 , while the subscales ranged between .70 to .78 . The corresponding coefficients for the South African sample were .93 and .70 to .79 .

\section{Procedure}

Survey forms containing the CDMSES-SF and asking questions about age, grade and gender were administered to students in Grades 8-12 across the two secondary schools that 
participated in the study. The classroom teachers who had been provided with instructions regarding the administration protocol administered the survey forms.

\section{Results}

To estimate the factor structure of the CDMSES-SF, two sets of exploratory factor analyses were conducted. These analyses were undertaken separately on the Australian and the South African samples. First, initial solutions were obtained using principal axis factoring with orthogonal (varimax) rotations. These analyses identified four factors on the Australian sample and four factors on the South African sample, with $54.87 \%$ and $53.84 \%$ of the variance accounted for in the respective samples. However, both solutions were factorially complex and not interpretable. For the Australian sample, 15 items had dual or triple loadings greater than .30 on more than one factor; for the South African sample, 11 items had dual or four-way loadings greater than .30 on more than one factor. These solutions are not reported in this paper. Second, the main factorial analyses were undertaken using principal axis factoring with oblique (direct oblimin) rotation. An oblique solution, which further simplifies the factors and variables by allowing the factors to be correlated was sought as the original scale was developed to measure related components of career decision-making self-efficacy. As the CDMSES-SF items all measure the same general construct, allowing the factors to be correlated presents no conceptual or interpretative problems. Factor loadings after oblique rotation are reported in Table 1. Eigenvalues, the percentage of variance explained, and the inter-factor correlations are presented in Table 2. 
Table 1

Principal axis factor estimates of the oblique (direct oblimin) factor loadings for the 25-item CDMSES-SF for the Australian and the South African samples.

\begin{tabular}{|c|c|c|c|c|c|c|c|c|c|}
\hline \multirow[b]{2}{*}{ Item } & \multicolumn{4}{|c|}{$\begin{array}{l}\text { Australian Sample } \\
\qquad N=563\end{array}$} & \multicolumn{5}{|c|}{$\begin{array}{l}\text { South African Sample } \\
\qquad N=416\end{array}$} \\
\hline & $\begin{array}{c}\text { Factor } \\
1\end{array}$ & $\begin{array}{l}\text { Factor } \\
2\end{array}$ & $\begin{array}{l}\text { Factor } \\
3\end{array}$ & $\begin{array}{l}\text { Factor } \\
\quad 4\end{array}$ & Item & $\begin{array}{c}\text { Factor } \\
1\end{array}$ & $\begin{array}{l}\text { Factor } \\
2\end{array}$ & $\begin{array}{c}\text { Factor } \\
3\end{array}$ & $\begin{array}{c}\text { Factor } \\
4\end{array}$ \\
\hline Q19 ${ }^{2}$ & .75 & -.17 & .12 & .03 & $\mathrm{Q} 4^{5}$ & .74 & -.03 & -.02 & -.08 \\
\hline Q $20^{3}$ & .73 & -.01 & -.02 & .06 & $\mathrm{Q} 1^{2}$ & .62 & .04 & .03 & -.10 \\
\hline $\mathrm{Q} 11^{3}$ & .60 & .06 & -.02 & .26 & $\mathrm{Q}^{1}$ & .62 & .04 & -.04 & .06 \\
\hline Q22 ${ }^{1}$ & .59 & .20 & .05 & -.30 & $\mathrm{Q} 7^{4}$ & .61 & -.08 & -.18 & .07 \\
\hline Q12 ${ }^{4}$ & .54 & .04 & .17 & .02 & $\mathrm{Q} 6^{3}$ & .56 & .06 & .08 & .32 \\
\hline Q $21^{4}$ & .52 & .16 & .11 & .04 & $\mathrm{Q} 10^{2}$ & .53 & .11 & -.10 & .04 \\
\hline Q8 ${ }^{5}$ & .47 & .27 & -.07 & .06 & $\mathrm{Q} 2^{3}$ & .50 & .11 & .14 & .32 \\
\hline $\mathrm{Q}^{1}$ & .44 & .30 & -.12 & .18 & $\mathrm{Q}^{4}{ }^{4}$ & .43 & -.00 & -.16 & .20 \\
\hline $\mathrm{Q}^{2} 3^{2}$ & .44 & .13 & .20 & -.18 & $\mathrm{Q}_{23}{ }^{2}$ & .41 & .06 & -.34 & -.21 \\
\hline Q24 & .42 & .14 & .21 & -.15 & $\mathrm{Q}^{5}$ & .40 & .09 & -.12 & .07 \\
\hline Q14 ${ }^{1}$ & .41 & .17 & .23 & -.04 & $\mathrm{Q} 15^{2}$ & .27 & .14 & -.24 & .11 \\
\hline $\mathrm{Q} 2^{3}$ & -.09 & .78 & .07 & -.02 & $\mathrm{Q} 17^{5}$ & -.11 & .80 & -.05 & -.04 \\
\hline $\mathrm{Q} 6^{3}$ & .07 & .69 & -.04 & .05 & Q $13^{5}$ & .07 & .73 & .00 & -.12 \\
\hline $\mathrm{Q}^{4}$ & .03 & .64 & .02 & .11 & Q18 1 & .14 & .41 & -.11 & .04 \\
\hline$Q 4^{5}$ & .05 & .52 & .20 & .10 & $\mathrm{Q} 16^{3}$ & .10 & .39 & .07 & .17 \\
\hline$Q 7^{4}$ & .38 & .49 & -.08 & .02 & $\mathrm{Q} 20^{3}$ & -.09 & .00 & -.69 & .29 \\
\hline $\mathrm{Q} 1^{2}$ & .07 & .39 & .18 & -.07 & $\mathrm{Q} 19^{2}$ & .10 & .01 & -.69 & -.13 \\
\hline $\mathrm{Q}^{1}$ & .32 & .35 & .05 & .06 & Q $24^{4}$ & -.01 & .15 & -.63 & .00 \\
\hline Q15 ${ }^{2}$ & .13 & .26 & .23 & .15 & $\mathrm{Q} 22^{1}$ & .07 & .07 & -.54 & .07 \\
\hline Q $17^{5}$ & -.02 & .01 & .74 & .09 & $\mathrm{Q} 21^{4}$ & .26 & -.03 & -.53 & -.04 \\
\hline Q13 ${ }^{5}$ & .05 & .05 & .61 & -.01 & $\mathrm{Q} 12^{4}$ & .20 & .06 & -.41 & .08 \\
\hline Q $25^{5}$ & .23 & .09 & .45 & -.00 & $\mathrm{Q} 25^{5}$ & .04 & .33 & -.38 & -.05 \\
\hline Q18 ${ }^{1}$ & .32 & .02 & .33 & .18 & Q14 ${ }^{1}$ & .26 & .23 & -.23 & .16 \\
\hline Q16 ${ }^{3}$ & -.00 & .16 & .22 & .39 & Q9 ${ }^{1}$ & .13 & -.02 & -.29 & .50 \\
\hline $\mathrm{Q} 10^{2}$ & .18 & .24 & .11 & .39 & $\mathrm{Q} 11^{3}$ & .17 & .04 & -.31 & .43 \\
\hline
\end{tabular}

Note $1 .{ }^{1}=$ items for Self-Appraisal subscale; ${ }^{2}=$ items for Occupational Information subscale; ${ }^{3}=$ items for Goal Selection subscale; ${ }^{4}=$ items for Planning subscale; ${ }^{5}=$ items for Problem Solving subscale.

From Table 1, it can be seen that a much simpler pattern of factor loadings has emerged than found for the initial orthogonal solution. Very few items have dual loadings greater than 
.30 on more than one factor (five in the Australian sample, and three in the South African sample), and no items load jointly on three or four factors. Item 15 has weak loadings in the Australian sample, and items 14 and 15 have weak loadings in the South African sample. This solution provides a much better approximation of simple structure. The factors themselves are similar over the two samples. The items contributing to Factor 1 in the Australian sample parallels those items contributing to Factor 3 in the South African sample and includes items from the five original subscales. Factor 2 in the Australian sample parallels Factor 1 in the South African sample, and again includes items from the five original subscales. Factor 3 in the Australian sample parallels Factor 2 in the South African sample, and primarily includes items from the original Problem Solving subscale. Factor 4 in both samples contains only two items. These items are not consistent over the two samples and Factor 4 should be considered unreliable and not interpretable (Tabachnick \& Fidell, 1996). Thus, the results of the Principal Axis factor analysis with oblique rotation have produced clearer and somewhat consistent results across the two high school student samples.

Despite these analyses identifying the most probable factor structure of the CDMSES-SF in each of the samples, it is important to note the pre-eminence of Factor 1 in both samples. In the Australian sample, Factor 1 is represented by 11 items and accounts for $40.03 \%$ of the $54.87 \%$ explained variance. In the South African sample, Factor 1 is also represented by 11 items and accounts for $37.46 \%$ of the $53.84 \%$ explained variance. Most importantly however, Factor 1 in the Australian sample does not parallel Factor 1 in the South African sample (Australian Factor 1 with 11 items parallels South African Factor 3 with eight items - seven of the eight items in the South African Factor 1 parallel the Australian Factor 1; South African Factor 1 parallels Australian Factor 2). This means that the main factor that emerged with the Australian sample is not measuring the same construct that is being measured by the main factor in the South African sample. Further, the items for each of the factors are drawn 
from across the five subscales of the measure. CDMSES-SF scores taken in Australia are likely to reflect a different construct to CDMSES-SF scores taken in South Africa.

Table 2

Eigenvalues, percentage of variance explained and the inter-factor correlations for the 25-item CDMSES-SF for the Australian and the South African samples.

\begin{tabular}{|c|c|c|c|c|c|c|c|c|c|c|c|c|}
\hline \multirow{3}{*}{ Factor } & \multicolumn{6}{|c|}{$\begin{array}{l}\text { Australian Sample } \\
\qquad N=563\end{array}$} & \multicolumn{6}{|c|}{$\begin{array}{l}\text { South African Sample } \\
\qquad N=416\end{array}$} \\
\hline & \multirow{2}{*}{$\begin{array}{l}\text { Eigen- } \\
\text { value }^{\#}\end{array}$} & \multirow{2}{*}{$\begin{array}{l}\text { Variance } \\
\text { explained }\end{array}$} & \multicolumn{4}{|c|}{ Correlations } & \multirow{2}{*}{$\begin{array}{l}\text { Eigen- } \\
\text { value }^{\#}\end{array}$} & \multirow{2}{*}{$\begin{array}{l}\text { Variance } \\
\text { explained }\end{array}$} & \multicolumn{4}{|c|}{ Correlations } \\
\hline & & & 1 & 2 & 3 & 4 & & & 1 & 2 & 3 & 4 \\
\hline 1 & 10.01 & 40.03 & - & .65 & .54 & .18 & 9.37 & 37.46 & - & .48 & -.62 & .38 \\
\hline 2 & 1.42 & 5.67 & & - & .51 & .31 & 1.63 & 6.62 & & - & -.45 & .19 \\
\hline 3 & 1.28 & 5.12 & & & - & .17 & 1.37 & 5.47 & & & - & -.25 \\
\hline 4 & 1.01 & 4.06 & & & & - & 1.10 & 4.38 & & & & - \\
\hline
\end{tabular}

Further cross-cultural differences can be identified in Table 2. The main factor in the Australian sample (Factor 1) is positively correlated with the two minor factors (Factors 2 and 3), while the two minor factors themselves are also positively correlated. In the South African sample, the main factor (Factor 1) is positively correlated with one of the minor factors (Factor 2), but negatively correlated with the other (Factor 3), and the two minor factors are negatively correlated. Thus, not only do the main factors across the two samples represent different constructs, these individual constructs have different relationships with their two minor factors respectively. Lastly, in relation to Table 2, the high correlations among the factors for both samples confirm the usefulness of the oblimin rather than orthogonal rotation as the optimum solution for the CDMSES-SF.

A number of points can be made from these analyses. First, no simple underlying structure emerged for the CDMSES-SF on either the Australian or South African sample. Second, the structures that did emerge differed quite markedly across the two national samples. Third, 
neither structure that emerged approximated the underlying structure reported by Betz et al. (1996) when they developed this short form of the scale on a US college sample. Given the divergent results from these exploratory analyses further cross-cultural equivalence analyses as suggested by Ben-Porath (1990) were not undertaken.

\section{Discussion}

On the positive side, this study was able to confirm that the CDMSES-SF has high internal reliability when used with high school age students across two national samples. The internal reliabilities using the full 25 items were both higher than .90 . The internal reliability coefficients for each of the five subscales across the two samples were all moderate to high. These results are consistent with reliability data reported on samples from other cultures and from other age groups (Betz et al, 1996; Betz \& Voyten, 1997; Gloria \& Hird, 1999).

However, despite these subscale reliabilities, the results of the exploratory factor analyses indicate that the CDMSES-SF cannot be utilised as a multifactorial scale, in the first instance with Australian or South African high school students, and possibly with high school students in general, although this latter recommendation needs to be tested across other cultures. Previous factor analytic studies have failed to support the five theorized factors of the CDMSES-SF with college samples (Betz et al, 1996; Betz \& Voyten, 1997; Gloria \& Hird, 1999), which has led to consistent recommendations that it only be used as a general measure of decision-making self-efficacy.

Previous research conducted with college samples has tested five and two factor solutions. The evidence from the present study conducted with high school students is that the CDMSES-SF reflects three interpretable factors, rather than the five originally intended or the two previously identified in a college student sample (Betz et al, 1996). For the present 
samples, items for the three identified factors were drawn from across the five subscales of the CDMSES-SF. For example, in Factor 1 from the Australian sample, all subscales contributed between one and three items to the factor. The evidence here, which is consistent with the earlier findings (e.g., Betz et al.), further supports the recommendation that the CDMSES-SF should be treated as a general measure of decision-making self-efficacy rather one that utilises the five theorised subscales.

While the present study found three factors for each of the two national samples, the factors for the Australian sample did not approximate those for the South African sample. Each sample produced a dominant factor that accounted for the majority of the variance explained, but these two main factors were not consistent across the samples. This is to say, the evidence from the exploratory analyses indicated that, even though the three factors in each sample were highly correlated, the factors differed cross-culturally. Additionally here, it should be reiterated that the structure for both the Australian and South African samples were inconsistent with the structure found for the US college samples (Betz et al., 1996). These findings have critical implications for cross-cultural self-efficacy research, and raise questions about the usefulness of the CDMSES-SF scale, even as a general measure of career decision-making self-efficacy with these national samples and age groups. First, research is needed to examine the cross-cultural equivalence of the CDMSES-SF (and by implication, the CDMSES) where it is used outside of the US. This applies to its use in non-US English speaking as well as non-English speaking countries. Second, cross-cultural equivalence also needs to be considered when researchers examine the decision-making self-efficacy across national boundaries outside of the US. Lonner (1981) outlined four types of cultural equivalence, being functional, conceptual, psychometric and linguistic. Based on the evidence from this study, which examined psychometric equivalence, cultural equivalence for 
the CDMSES-SF between US and Australia/South Africa and between Australia and South Africa, cannot be assumed.

It can also be concluded that a number of items in the CDMSES-SF may be redundant and that a more parsimonious measure of the present scale is possible. Lastly, the CDMSES and CDMSES-SF were constructed to reflect the five career choice competencies that Crites (1961) proposed as relevant for the career decision-making process. The results from this study indicate that not all five competencies are sufficiently reflected in the CDMSES-SF, which argues for a revision of the scale itself. 


\section{References}

Albion, M. J. (2000). Developing and validating a model of career decision making. Unpublished doctoral dissertation, University of Southern Queensland, Australia.

Bandura, A. (1986). Social foundations of thought and action: A social cognitive theory. Englewood Cliffs, NJ: Prentice-Hall.

Ben-Porath, Y. S. (1990). Cross-cultural assessment of personality: The case of replicatory factor analysis. In J. N. Butcher \& C. D. Spielberger (Eds.). Recent advances in personality assessment: Vol. 8 (pp. 27 - 48). Hilldale, NJ: Erlbaum.

Betz, N. E., \& Hackett, G. (1986). Applications of self-efficacy theory to understanding career choice behavior. Journal of Social and Clinical Psychology, 4, 279-289.

Betz, N. E., \& Klein, K. L. (1996). Relationships among career self-efficacy, generalized self-efficacy, and global self-esteem. Journal of Career Assessment, 4, 47-57.

Betz, N. E., Klein, K. L., \& Taylor, K. M. (1996). Evaluation of a short form of the Career Decision-Making Self-Efficacy Scale. Journal of Career Assessment, 4, 47-57.

Betz, N. E., \& Voyten, K. K. (1997). Efficacy and outcome expectations influence career exploration and decidedness. The Career Development Quarterly, 46, 179-189.

Blustein, D. L. (1989). The role of goal instability and career self-efficacy in the career exploration process. Journal of Vocational Behavior, 35, 194-203.

Crites, J. O. (1961). A model for the measurement of vocational maturity. Journal of Counseling Psychology, 8, 255-259.

Gati, I., Osipow, S. H., \& Fassa, N. (1994). The scale structure of multi-scale measures: Application of the split-scale method to the Task Specific Occupational Self-Efficacy Scale and the Career Decision-Making Self-Efficacy Scale. Journal of Career Assessment, 2, 384387. 
Gianakos, I. (1999). Patterns of career choice and career decision-making self-efficacy. Journal of Vocational Behavior, 54, 244-258.

Gloria, A. M., \& Hird, J. S. (1999). Influences of ethnic and nonethnic variables on the career decision-making self-efficacy of college students. Career Development Quarterly, 48, 157-174.

Hackett, G., \& Lent, R. (1992). Theoretical advances in career psychology. In S. Brown \& R. Lent (Eds.), Handbook of counseling psychology (2 ${ }^{\text {nd }}$ ed.). New York: Wiley.

Helms, J. E. (1992). Why is there no study of cultural equivalence in standardized cognitive ability testing? American Psychologist, 1, 185-216.

Holland, J. L., Johnston, J. A., \& Asama, N. F. (1993). The Vocational Identity Scale: A diagnostic and treatment tool. Journal of Career Assessment, 1, 1-12.

Kraus, L. J., \& Hughey, K. F. (1999). The impact of an intervention on career decisionmaking self-efficacy and career indecision. Professional School Counseling, 2, 384-390.

Lent, R. W., Brown, S. D., \& Hackett, G. (1994). Toward a unifying social cognitive theory of career and academic interest, choice, and performance. Journal of Vocational Behavior, 45, 79-122.

Lent, R. W., \& Hackett, G. (1987). Career self-efficacy: Empirical status and future directions. Journal of Vocational Behavior, 30, 347-382.

Lonner, W. J. (1981). Psychological tests and intercultural counseling. In P. B. Pedersen, J. G. Draguns, E. J. Lonner, \& J. E. Trimble (Eds.), Counseling across cultures. Honolulu: East-West Centre and University of Hawaii.

Luzzo, D. A. (1995). The relative contributions of self-efficacy and locus of control to the prediction of career maturity. Journal of College Student Development, 36, 61-66.

Luzzo, D. A. (1996). A psychometric evaluation of the Career Decision-Making SelfEfficacy Scale. Journal of Counseling and Development, 74, 276-279. 
Luzzo, D. A., Hitchings, W. E., Retish, P., \& Shoemaker, A. (1999). Evaluating differences in college students' career decision making on the basis of disability status. Career Development Quarterly, 48, 142-156.

Osipow, S. H. (1991). Developing instruments for use in counseling. Journal of Counseling \& Development, 70, 322-326.

Peterson, S. L., \& delMas, R. C. (1998). The component structure of career decisionmaking self-efficacy for underprepared college students. Journal of Career Development, 24, 209-225.

Robbins, S. B. (1985). Validity estimates for the Career Decision-Making Self-Efficacy Scale. Measurement and Evaluation in Counseling and Development, 18, 64-71.

Solberg, V. S., Good, G. E., Nord, D., Holm, C., Hohner, R., Zima, N., Heffernan, M., \& Malen, A. (1994). Assessing career search expectations: Development and validation of the Career Search Efficacy Scale. Journal of Career Assessment, 2, 111-123.

Tabachnick, B. G., \& Fidell, L. S. (1996). Using multivariate statistics (3 ${ }^{\text {rd }}$ Edition). Northbridge, Cal.: Harper Collins.

Taylor, K. M., \& Betz, N. E. (1983). Applications of self-efficacy theory to the understanding and treatment of career indecision. Journal of Vocational Behavior, 22, 63-81.

Taylor, K. M., \& Popma, J. (1990). An examination of the relationships among career decision-making self-efficacy, career salience, locus of control, and vocational indecision. Journal of Vocational Behavior, 37, 17-31.

Watson, M. B., Brand, H. J., Stead, G. B., \& Ellis, R. R. (2001). Confirmatory factor analysis of the Career Decision-Making Self-Efficacy Scale among South African university students. Journal of Industrial Psychology, 27(1), 43-46. 